doi: $10.2306 /$ scienceasia1513-1874.2014.40.301

\title{
Convergence of three-step iterations for total asymptotically nonexpansive mappings in uniformly convex Banach spaces
}

\author{
Zhanfei Zuo \\ Department of Mathematics and Statistics, Chongqing Three Gorges University, Wanzhou 404100 China
}

e-mail: zuozhanfei@139.com

Received 27 May 2013

Accepted 20 Dec 2013

\begin{abstract}
In this paper, we study a three-step iterative scheme for total asymptotically nonexpansive mappings in uniformly convex Banach spaces. As an application, several convergence theorems of this scheme are established for total asymptotically nonexpansive mappings. The results obtained in this paper are an extension and refinement of some previous results.
\end{abstract}

KEYWORDS: three-step mean value iterative scheme, uniformly $L$-Lipschitzian condition, Opial's condition, convergence theorem

\section{INTRODUCTION}

Noor introduced a three-step iterative scheme and studied the approximate solutions of variational inclusion in Hilbert spaces ${ }^{1}$. Glowinski and Le Tallec used three-step iterative schemes to find the approximate solutions of the elastoviscoplasticity problem, liquid crystal theory, and eigenvalue computation ${ }^{2}$. It has been shown that the three-step iterative scheme gives better numerical results than the two-step and onestep approximate iterations ${ }^{2}$. Haubruge et al studied the convergence analysis of three-step schemes of Glowinski and Le Tallec and applied these schemes to obtain new splitting-type algorithms for solving variational inequalities, separable convex programming and minimization of a sum of convex functions ${ }^{3}$. They also proved that three-step iterations lead to highly parallelized algorithms under certain conditions. Thus three-step scheme plays an important and significant part in solving various problems, which arise in pure and applied sciences. $\mathrm{Xu}$ and Noor introduced and studied a three-step scheme to approximate fixed point of asymptotically nonexpansive mappings in a Banach space $^{4}$. Suantai defined a new three-step iteration which is an extension of $\mathrm{Xu}$ and Noor iterations and gave some weak and strong convergence theorems of the iterations for asymptotically nonexpansive mappings in a uniformly convex Banach space ${ }^{5}$. Very recently, Nilsrakoo and Saejung defined a new threestep iterations which is an extension of Noor iterations and gave some weak and strong convergence theo- rems of the modified Noor iterations for asymptotically nonexpansive mappings in Banach space ${ }^{6}$. The scheme is defined as follows.

Algorithm 1 Let $C$ be a nonempty closed subset of a real Banach space $X$ and $T: C \rightarrow C$ be a given mapping. For a given $x_{1} \in C$, compute sequences $\left\{z_{n}\right\},\left\{y_{n}\right\},\left\{x_{n}\right\}$ by the iterative scheme

$$
\begin{aligned}
x_{n+1}=\alpha_{n} T^{n} y_{n}+ & \beta_{n} T^{n} z_{n}+\gamma_{n} T^{n} x_{n} \\
& +\left(1-\alpha_{n}-\beta_{n}-\gamma_{n}\right) x_{n}
\end{aligned}
$$

where $z_{n}=a_{n} T^{n} x_{n}+\left(1-a_{n}\right) x_{n} ; y_{n}=b_{n} T^{n} z_{n}+$ $c_{n} T^{n} x_{n}+\left(1-b_{n}-c_{n}\right) x_{n}$; and $\left\{a_{n}\right\},\left\{b_{n}\right\},\left\{c_{n}\right\}$, $\left\{b_{n}+c_{n}\right\},\left\{\alpha_{n}\right\},\left\{\beta_{n}\right\},\left\{\gamma_{n}\right\}$, and $\left\{\alpha_{n}+\beta_{n}+\gamma_{n}\right\}$ are appropriate sequences in $[0,1]$.

The iterative scheme (1) is called the three-step mean value iterative scheme. It is clear that the three-step mean value iterative scheme includes the modified Noor iterations, furthermore, the modified Noor iterations include Mann iterations, Ishikawa iterations, and original Noor iterations as special cases. It is our purpose to establish a few weak and strong convergence theorems of the three-step mean value iterative for $\left(\left\{\mu_{n}\right\},\left\{\nu_{n}\right\}, \zeta\right)$-total asymptotically nonexpansive mapping in a uniformly convex Banach space. Our results extend and improve the corresponding results announced by $\mathrm{Xu}$ and Noor ${ }^{4}$, Suantai ${ }^{5}$, Nilsrakoo and Saejung 6,7. 


\section{PRELIMINARIES}

The asymptotically nonexpansive mapping is defined by Gobel and Kirk ${ }^{8}$.

Definition 1 Let $C$ be bounded subset of $X$, a mapping $T: X \rightarrow X$ is called asymptotically nonexpansive if there exists a sequence $\left\{k_{n}\right\}$ of positive real numbers with $k_{n} \rightarrow 1$ for which

$$
\left\|T^{n} x-T^{n} y\right\| \leqslant k_{n}\|x-y\| \quad \text { for all } x, y \in X .
$$

Definition $2 T$ is said to be uniformly $L$-Lipschitzian, if there exists a constant $L>0$ such that

$\left\|T^{n} x-T^{n} y\right\| \leqslant L\|x-y\|$ for all $n \geqslant 1, x, y \in X$.

Chang et al recently introduced the concept of total asymptotically nonexpansive mappings in metric spaces and proved the demiclosed principle for this kind of mapping ${ }^{9}$. Similarly, we can get the total asymptotically nonexpansive mappings in Banach space $X$.

Definition 3 A mapping $T: X \rightarrow X$ is said to be $\left(\left\{\mu_{n}\right\},\left\{\nu_{n}\right\}, \zeta\right)$-total asymptotically nonexpansive, if there exist nonnegative sequences $\left\{\mu_{n}\right\},\left\{\nu_{n}\right\}$ with $\mu_{n} \rightarrow 0, \nu_{n} \rightarrow 0$ and a strictly increasing continuous function $\zeta:[0, \infty) \rightarrow[0, \infty)$ with $\zeta(0)=0$ such that

$$
\left\|T^{n} x-T^{n} y\right\| \leqslant\|x-y\|+\nu_{n} \zeta(\|x-y\|)+\mu_{n}
$$

for all $n \geqslant 1, x, y \in X$.

Remark 1 From the above definitions, it is to know that each nonexpansive mapping is an asymptotically nonexpansive mapping with sequence $\left\{k_{n}=1\right\}$ and each asymptotically nonexpansive mapping is a $\left(\left\{\mu_{n}\right\},\left\{\nu_{n}\right\}, \zeta\right)$-total asymptotically nonexpansive mapping with $\mu_{n}=0, \nu_{n}=k_{n}-1$ for all $n \geqslant 1$ and $\zeta(t)=t$ for all $t \geqslant 0$.

\section{MAIN RESULTS}

Lemma 1 (Ref. 10) Let $\left\{a_{n}\right\},\left\{\lambda_{n}\right\}$ and $\left\{c_{n}\right\}$ be sequences of nonnegative numbers such that

$$
a_{n+1} \leqslant\left(1+\lambda_{n}\right) a_{n}+c_{n} \text { for all } n \geqslant 1 .
$$

If $\sum_{n=1}^{\infty} \lambda_{n}<\infty$ and $\sum_{n=1}^{\infty} c_{n}<\infty$, then $\lim _{n} a_{n}$ exists. If there exists a subsequence of $\left\{a_{n}\right\}$ which converges to 0 , then $\lim _{n} a_{n}=0$.

Lemma 2 Let $X$ be a real Banach space, $C$ be a nonempty closed convex subset of $X$, and $T: C \rightarrow C$ be a $\left(\left\{\mu_{n}\right\},\left\{\nu_{n}\right\}, \zeta\right)$-total asymptotically nonexpansive mapping. Let $\left\{a_{n}\right\},\left\{b_{n}\right\},\left\{\alpha_{n}\right\}$ be sequences of real numbers in $[0,1]$, and $\left\{x_{n}\right\}$ be a sequence in $C$ defined by Algorithm 1. If (i) $\sum_{n=1}^{\infty} \nu_{n}<\infty ; \sum_{n=1}^{\infty} \mu_{n}<\infty$, and

(ii) there exists a constant $M^{*}>0$ such that $\zeta(r) \leqslant M^{*} r$ for all $r \geqslant 0$, then $\lim _{n}\left\|x_{n}-p\right\|$ exists for each fixed point $p$ of $T$.

Proof: Let $p$ be a fixed point of $T$. From the definition of $\left\{y_{n}\right\}$ and $\left\{z_{n}\right\}$ in Algorithm 1, and that $T^{n} p=p$, we have

$$
\begin{aligned}
\left\|z_{n}-p\right\| \leqslant & a_{n}\left\|T^{n} x_{n}-T^{n} p\right\|+\left(1-a_{n}\right)\left\|x_{n}-p\right\| \\
\leqslant & a_{n}\left[\left\|x_{n}-p\right\|+\nu_{n} \zeta\left(\left\|x_{n}-p\right\|\right)+\mu_{n}\right] \\
& +\left(1-a_{n}\right)\left\|x_{n}-p\right\| \\
\leqslant & \left(1+\nu_{n} M^{*}\right)\left\|x_{n}-p\right\|+\mu_{n} .
\end{aligned}
$$

$$
\begin{aligned}
\| y_{n}- & p \| \\
\leqslant & b_{n}\left\|T^{n} z_{n}-T^{n} p\right\|+c_{n}\left\|T^{n} x_{n}-T^{n} p\right\| \\
& +\left(1-b_{n}-c_{n}\right)\left\|x_{n}-p\right\| \\
\leqslant & b_{n}\left[\left\|z_{n}-p\right\|+\nu_{n} \zeta\left(\left\|z_{n}-p\right\|\right)+\mu_{n}\right] \\
& +c_{n}\left[\left\|x_{n}-p\right\|+\nu_{n} \zeta\left(\left\|x_{n}-p\right\|\right)+\mu_{n}\right] \\
& +\left(1-b_{n}-c_{n}\right)\left\|x_{n}-p\right\| \\
\leqslant & b_{n}\left[\left(1+\nu_{n} M^{*}\right)\left\|x_{n}-p\right\|+\mu_{n}\right. \\
& \left.+\nu_{n} M^{*}\left(\left(1+\nu_{n} M^{*}\right)\left\|x_{n}-p\right\|+\mu_{n}\right)+\mu_{n}\right] \\
& +c_{n}\left[\left\|x_{n}-p\right\|+v_{n} M^{*}\left\|x_{n}-p\right\|+\mu_{n}\right] \\
& +\left(1-b_{n}-c_{n}\right)\left\|x_{n}-p\right\| \\
\leqslant & \left(1+3 \nu_{n} M^{*}+\left(\nu_{n} M^{*}\right)^{2}\right)\left\|x_{n}-p\right\| \\
& +\left(\nu_{n} M^{*}+3\right) \mu_{n} .
\end{aligned}
$$

From (1), (2), (3), we get

$$
\begin{aligned}
\| & x_{n+1}-p \| \\
\leqslant & \alpha_{n}\left\|T^{n} y_{n}-T^{n} p\right\|+\beta_{n}\left\|T^{n} z_{n}-T^{n} p\right\| \\
& +\gamma_{n}\left\|T^{n} x_{n}-T^{n} p\right\| \\
& +\left(1-\alpha_{n}-\beta_{n}-\gamma_{n}\right)\left\|x_{n}-p\right\| \\
\leqslant & \alpha_{n}\left[\left(1+\nu_{n} M^{*}\right)\left\|y_{n}-p\right\|+\mu_{n}\right] \\
& +\beta_{n}\left[\left(1+\nu_{n} M^{*}\right)\left\|z_{n}-p\right\|+\mu_{n}\right] \\
& +\gamma_{n}\left[\left(1+v_{n} M^{*}\right)\left\|x_{n}-p\right\|+\mu_{n}\right] \\
& +\left(1-\alpha_{n}-\beta_{n}-\gamma_{n}\right)\left\|x_{n}-p\right\| \\
\leqslant & \left(1+7 \nu_{n} M^{*}+5\left(\nu_{n} M^{*}\right)^{2}+\left(\nu_{n} M^{*}\right)^{3}\right)\left\|x_{n}-p\right\| \\
& +\left(7+5 \nu_{n} M^{*}+\left(\nu_{n} M^{*}\right)^{2}\right) \mu_{n} .
\end{aligned}
$$

In Lemma 1, take $a_{n}=\left\|x_{n}-p\right\|, \lambda_{n}=7 \nu_{n} M^{*}+$ $5\left(\nu_{n} M^{*}\right)^{2}+\left(\nu_{n} M^{*}\right)^{3}$ and $c_{n}=\left(7+5 \nu_{n} M^{*}+\right.$ $\left.\left(\nu_{n} M^{*}\right)^{2}\right) \mu_{n}$, then all conditions in Lemma 1 are satisfied. The conclusion is obtained from Lemma 1 immediately.

Lemma 3 (Ref. 7) Let $\left\{x_{n}\right\},\left\{y_{n}\right\}$ and $\left\{z_{n}\right\}$ be sequences in a uniformly convex Banach space $X$. 
Suppose that $\left\{\alpha_{n}\right\},\left\{\beta_{n}\right\}$ and $\left\{\gamma_{n}\right\}$ are sequences in $[0,1]$ with $\alpha_{n}+\beta_{n}+\gamma_{n}=1, \limsup _{n}\left\|x_{n}\right\| \leqslant$ $d, \lim \sup _{n}\left\|y_{n}\right\| \leqslant d, \limsup _{n}\left\|z_{n}\right\| \leqslant d$ and $\lim _{n}\left\|\alpha_{n} x_{n}+\beta_{n} y_{n}+\gamma_{n} z_{n}\right\|=d$. If $\liminf _{n} \alpha_{n}>$ 0 and $\liminf _{n} \beta_{n}>0$, then $\lim _{n}\left\|x_{n}-y_{n}\right\|=0$.

In fact, we can also obtain the following Lemma.

Lemma 4 Let $\left\{x_{n}\right\},\left\{y_{n}\right\},\left\{z_{n}\right\}$ and $\left\{u_{n}\right\}$ be sequences in a uniformly convex Banach space $X$. Suppose that $\left\{\alpha_{n}\right\},\left\{\beta_{n}\right\},\left\{\gamma_{n}\right\}$ and $\left\{\delta_{n}\right\}$ are sequences in $[0,1]$ with $\alpha_{n}+\beta_{n}+\gamma_{n}+$ $\delta_{n}=1, \quad \lim \sup _{n}\left\|x_{n}\right\| \leqslant d, \lim \sup _{n}\left\|y_{n}\right\| \leqslant$ $d, \lim \sup _{n}\left\|z_{n}\right\| \leqslant d, \lim \sup _{n}\left\|u_{n}\right\| \leqslant d$ and $\lim _{n}\left\|\alpha_{n} x_{n}+\beta_{n} y_{n}+\gamma_{n} z_{n}+\delta_{n} u_{n}\right\|=d$. If $\liminf \alpha_{n}>0$ and $\liminf _{n} \beta_{n}>0$, then $\lim _{n}\left\|x_{n}-y_{n}\right\|=0$.

Proof: The proof is similar to that of Lemma 3; therefore, the detail is omitted.

Lemma 5 Let $X$ be a real Banach space, $C$ be a nonempty closed convex subset of $X$, and $T: C \rightarrow C$ be an $\left(\left\{\mu_{n}\right\},\left\{\nu_{n}\right\}, \zeta\right)$-total asymptotically nonexpansive mapping, $\left\{x_{n}\right\}$ be a sequence in $C$ defined by Algorithm 1. If

(i) $\sum_{n=1}^{\infty} \nu_{n}<\infty ; \sum_{n=1}^{\infty} \mu_{n}<\infty$,

(ii) there exists a constant $M^{*}>0$ such that $\zeta(r) \leqslant$ $M^{*} r$ for all $r \geqslant 0$, and

(iii) $\lim _{n}\left\|T^{n} x_{n}-x_{n}\right\|=0$,

then $\lim _{n}\left\|T x_{n}-x_{n}\right\|=0$.

Proof: From the conditions in the Lemma, we get

$$
\begin{aligned}
&\left\|T^{n} z_{n}-x_{n}\right\| \leqslant\left\|T^{n} z_{n}-T^{n} x_{n}\right\|+\left\|T^{n} x_{n}-x_{n}\right\| \\
& \leqslant\left(1+\nu_{n} M^{*}\right)\left\|z_{n}-x_{n}\right\|+\mu_{n} \\
&+\left\|T^{n} x_{n}-x_{n}\right\| \\
& \leqslant\left(1+\nu_{n} M^{*}\right) a_{n}\left\|T^{n} x_{n}-x_{n}\right\|+\mu_{n} \\
&+\left\|T^{n} x_{n}-x_{n}\right\| \rightarrow 0, \\
&\left\|T^{n} y_{n}-x_{n}\right\| \leqslant\left\|T^{n} y_{n}-T^{n} x_{n}\right\|+\left\|T^{n} x_{n}-x_{n}\right\| \\
& \leqslant\left(1+\nu_{n} M^{*}\right)\left[\left\|y_{n}-x_{n}\right\|+\mu_{n}\right] \\
&+\left\|T^{n} x_{n}-x_{n}\right\| \\
& \leqslant\left(1+\nu_{n} M^{*}\right)\left[b_{n}\left\|T^{n} z_{n}-x_{n}\right\|\right. \\
&\left.+c_{n}\left\|T^{n} x_{n}-x_{n}\right\|+\mu_{n}\right] \\
&+\left\|T^{n} x_{n}-x_{n}\right\| \rightarrow 0, \\
& \| x_{n+1}- T^{n} x_{n+1} \| \\
& \leqslant\left\|x_{n+1}-x_{n}\right\|+\left\|T^{n} x_{n+1}-T^{n} x_{n}\right\| \\
&+\left\|T^{n} x_{n}-x_{n}\right\| \\
& \leqslant\left(2+\nu_{n} M^{*}\right)\left\|x_{n+1}-x_{n}\right\|+\mu_{n} \\
&+\left\|T^{n} x_{n}-x_{n}\right\| \\
& \leqslant\left(2+\nu_{n} M^{*}\right)\left[\alpha_{n}\left\|T^{n} y_{n}-x_{n}\right\|\right.
\end{aligned}
$$

$$
\begin{aligned}
& \left.+\beta_{n}\left\|T^{n} z_{n}-x_{n}\right\|+\gamma_{n}\left\|T^{n} x_{n}-x_{n}\right\|\right] \\
& +\mu_{n}+\left\|T^{n} x_{n}-x_{n}\right\| \rightarrow 0 .
\end{aligned}
$$

Then

$$
\begin{aligned}
& \left\|x_{n+1}-T x_{n+1}\right\| \\
& \quad \leqslant\left\|x_{n+1}-T^{n+1} x_{n+1}\right\|+\left\|T^{n+1} x_{n+1}-T x_{n+1}\right\| \\
& \quad \leqslant\left\|x_{n+1}-T^{n+1} x_{n+1}\right\| \\
& \quad+\left(1+\nu_{n} M^{*}\right)\left\|x_{n+1}-T^{n} x_{n+1}\right\|+\mu_{n} \rightarrow 0 .
\end{aligned}
$$

Lemma 6 Let $X$ be a real Banach space, $C$ be a nonempty closed convex subset of $X$, and $T: C \rightarrow C$ be a $\left(\left\{\mu_{n}\right\},\left\{\nu_{n}\right\}, \zeta\right)$-total asymptotically nonexpansive mapping. Let $\left\{x_{n}\right\}$ be a sequence in $C$ defined by Algorithm 1 and the parameters satisfy one of the following control conditions:

(i) $\lim \inf _{n} \alpha_{n}>0$ and one of the following holds:

(a) $0<\liminf _{n} \beta_{n} \leqslant \lim \sup _{n}\left(\alpha_{n}+\beta_{n}+\gamma_{n}\right)<1$ and $\lim \sup _{n} c_{n}<1$;

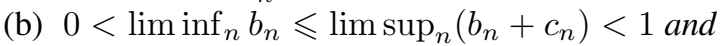
$\lim \sup _{n} a_{n}<1$;

(c) $0<\liminf { }_{n} c_{n} \leqslant \lim \sup _{n}\left(b_{n}+c_{n}\right)<1$;

(ii) $0<\liminf _{n} \beta_{n}$ and

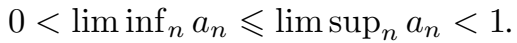

Then $\lim _{n}\left\|x_{n}-T x_{n}\right\|=0$.

Proof: Let $p$ be a fixed point of $T$. From Lemma 2, $\lim _{n}\left\|x_{n}-p\right\|=d$ for some $d \geqslant 0$. It follows from (1) that

$$
\begin{aligned}
d=\lim _{n} \| & x_{n+1}-p \| \\
=\lim _{n} \| & \alpha_{n}\left(T^{n} y_{n}-p\right)+\beta_{n}\left(T^{n} z_{n}-p\right) \\
& +\gamma_{n}\left(T^{n} x_{n}-p\right) \\
& +\left(1-\alpha_{n}-\beta_{n}-\gamma_{n}\right)\left(x_{n}-p\right) \| .
\end{aligned}
$$

From (2) and (3), we get

$$
\begin{aligned}
\left\|z_{n}-p\right\| \leqslant & \left(1+\nu_{n} M^{*}\right)\left\|x_{n}-p\right\|+\mu_{n} \\
\left\|y_{n}-p\right\| \leqslant & \left(1+3 \nu_{n} M^{*}+\left(\nu_{n} M^{*}\right)^{2}\right)\left\|x_{n}-p\right\| \\
& +\left(\nu_{n} M^{*}+3\right) \mu_{n} .
\end{aligned}
$$

From (5) and (6), we have

$$
\begin{aligned}
& \lim \sup _{n}\left\|T^{n} x_{n}-p\right\|=\lim \sup _{n}\left\|T^{n} x_{n}-T^{n} p\right\| \\
& \quad \leqslant \lim \sup _{n}\left[\left(1+\nu_{n} M^{*}\right)\left\|x_{n}-p\right\|+\mu_{n}\right]=d, \\
& \lim \sup _{n}\left\|T^{n} y_{n}-p\right\|=\lim \sup _{n}\left\|T^{n} y_{n}-T^{n} p\right\| \\
& \quad \leqslant \lim \sup _{n}\left[\left(1+\nu_{n} M^{*}\left\|y_{n}-p\right\|\right)+\mu_{n}\right] \leqslant d,
\end{aligned}
$$

and

$\limsup \sup _{n}\left\|T^{n} z_{n}-p\right\|=\lim \sup _{n}\left\|T^{n} z_{n}-T^{n} p\right\|$ $\leqslant \lim \sup _{n}\left[\left(1+\nu_{n} M^{*}\right)\left\|z_{n}-p\right\|+\mu_{n}\right] \leqslant d$. 
From (4), the condition (i-a), and Lemma 4, we have

$$
\lim _{n}\left\|T^{n} y_{n}-x_{n}\right\|=\lim _{n}\left\|T^{n} z_{n}-x_{n}\right\|=0 .
$$

Notice that

$$
\begin{aligned}
& \left\|T^{n} x_{n}-x_{n}\right\| \\
& \leqslant \\
& \leqslant T^{n} x_{n}-T^{n} y_{n}\|+\| T^{n} y_{n}-x_{n} \| \\
& \leqslant\left(1+\nu_{n} M^{*}\right)\left\|x_{n}-y_{n}\right\|+\mu_{n}+\left\|T^{n} y_{n}-x_{n}\right\| \\
& \leqslant \\
& \quad b_{n}\left\|T^{n} z_{n}-x_{n}\right\|+c_{n}\left\|T^{n} x_{n}-x_{n}\right\| \\
& \quad+\nu_{n} M^{*}\left\|x_{n}-y_{n}\right\|+\mu_{n}+\left\|T^{n} y_{n}-x_{n}\right\| .
\end{aligned}
$$

From the condition (i-a), $\lim \sup _{n} c_{n}<1$,

$$
\lim _{n}\left\|T^{n} x_{n}-x_{n}\right\|=0 .
$$

From the condition (i-b), (1), (4) and (5), we get

$$
\begin{aligned}
& \left\|x_{n+1}-p\right\| \\
& \leqslant \\
& \quad \alpha_{n}\left[\left(1+\nu_{n} M^{*}\right)\left\|y_{n}-p\right\|+\mu_{n}\right] \\
& \quad+\beta_{n}\left[\left(1+\nu_{n} M^{*}\right)\left\|z_{n}-p\right\|+\mu_{n}\right] \\
& \quad+\gamma_{n}\left[\left(1+\nu_{n} M^{*}\right)\left\|x_{n}-p\right\|+\mu_{n}\right] \\
& \quad+\left(1-\alpha_{n}-\beta_{n}-\gamma_{n}\right)\left\|x_{n}-p\right\| \\
& \leqslant \\
& \quad \alpha_{n}\left[\left(1+\nu_{n} M^{*}\right)\left\|y_{n}-p\right\|+\mu_{n}\right] \\
& \quad+\beta_{n}\left[\left(1+\nu_{n} M^{*}\right)\left(\left(1+\nu_{n} M^{*}\right)\left\|x_{n}-p\right\|+\mu_{n}\right)\right. \\
& \left.\quad+\mu_{n}\right]+\gamma_{n}\left[\left(1+\nu_{n} M^{*}\right)\left\|x_{n}-p\right\|+\mu_{n}\right] \\
& \quad+\left(1-\alpha_{n}-\beta_{n}-\gamma_{n}\right)\left\|x_{n}-p\right\| .
\end{aligned}
$$

That is

$$
\begin{gathered}
\frac{1}{\alpha_{n}}\left(\left\|x_{n+1}-p\right\|-\left\|x_{n}-p\right\|\right)+\left\|x_{n}-p\right\| \\
\leqslant\left(1+\nu_{n} M^{*}\right)\left\|y_{n}-p\right\|+\mu_{n} \\
\left.+\frac{\beta_{n}}{\alpha_{n}}\left(2+\nu_{n} M^{*}\right)\left[\nu_{n} M^{*}\left\|x_{n}-p\right\|+\mu_{n}\right)\right] \\
+\frac{\gamma_{n}}{\alpha_{n}}\left[\nu_{n} M^{*}\left\|x_{n}-p\right\|+\mu_{n}\right] .
\end{gathered}
$$

From $\liminf \operatorname{in}_{n} \alpha_{n}>0$, we get

$$
d=\lim _{n}\left\|x_{n}-p\right\| \leqslant \lim _{n} \inf \left\|y_{n}-p\right\| .
$$

This implies from (6) that

$$
\begin{gathered}
d=\lim _{n}\left\|y_{n}-p\right\| \\
=\lim _{n} \| b_{n}\left(T^{n} z_{n}-p\right)+c_{n}\left(T^{n} x_{n}-p\right) \\
\quad+\left(1-b_{n}-c_{n}\right)\left(x_{n}-p\right) \| .
\end{gathered}
$$

By Lemma 3, we get

$$
\lim _{n}\left\|T^{n} z_{n}-x_{n}\right\|=0 .
$$

Thus

$$
\begin{aligned}
\left\|T^{n} x_{n}-x_{n}\right\| \leqslant & \left\|T^{n} x_{n}-T^{n} z_{n}\right\|+\left\|T^{n} z_{n}-x_{n}\right\| \\
\leqslant & \left(1+\nu_{n} M^{*}\right)\left\|x_{n}-z_{n}\right\|+\mu_{n} \\
& +\left\|T^{n} z_{n}-x_{n}\right\| \\
\leqslant & a_{n}\left\|T^{n} x_{n}-x_{n}\right\|+\nu_{n} M^{*}\left\|x_{n}-z_{n}\right\| \\
& +\mu_{n}+\left\|T^{n} z_{n}-x_{n}\right\| .
\end{aligned}
$$

Since $\lim _{n} \sup a_{n}<1$,

$$
\lim _{n}\left\|T^{n} x_{n}-x_{n}\right\|=0 .
$$

By the condition (i-c), Lemma 3 and (7), we have

$$
\lim _{n}\left\|T^{n} x_{n}-x_{n}\right\|=0 .
$$

Finally, we will prove (ii)

$$
\begin{aligned}
&\left\|x_{n+1}-p\right\| \\
& \leqslant \alpha_{n}\left[\left(1+\nu_{n} M^{*}\right)\left\|y_{n}-p\right\|+\mu_{n}\right] \\
&+\beta_{n}\left[\left(1+\nu_{n} M^{*}\right)\left\|z_{n}-p\right\|+\mu_{n}\right] \\
&+\gamma_{n}\left[\left(1+\nu_{n} M^{*}\right)\left\|x_{n}-p\right\|+\mu_{n}\right] \\
&+\left(1-\alpha_{n}-\beta_{n}-\gamma_{n}\right)\left\|x_{n}-p\right\| \\
& \leqslant \alpha_{n}\left[\left(1+3 \nu_{n} M^{*}+\left(\nu_{n} M^{*}\right)^{2}\right)\left\|x_{n}-p\right\|\right. \\
&\left.+\left(\nu_{n} M^{*}+3\right) \mu_{n}+\nu_{n} M^{*}\left\|y_{n}-p\right\|+\mu_{n}\right] \\
&+\beta_{n}\left[\left(1+\nu_{n} M^{*}\right)\left\|z_{n}-p\right\|+\mu_{n}\right] \\
&+\gamma_{n}\left[\left(1+\nu_{n} M^{*}\right)\left\|x_{n}-p\right\|+\mu_{n}\right] \\
&+\left(1-\alpha_{n}-\beta_{n}-\gamma_{n}\right)\left\|x_{n}-p\right\| .
\end{aligned}
$$

That is

$$
\begin{gathered}
\frac{1}{\beta_{n}}\left(\left\|x_{n+1}-p\right\|-\left\|x_{n}-p\right\|\right)+\left\|x_{n}-p\right\| \\
\leqslant\left(1+\nu_{n} M^{*}\right)\left\|z_{n}-p\right\|+\mu_{n} \\
+\frac{\alpha_{n}}{\beta_{n}}\left[\left(3+\nu_{n} M^{*}\right)\left(\nu_{n} M^{*}\left\|x_{n}-p\right\|+\mu_{n}\right)\right. \\
\left.+\nu_{n} M^{*}\left\|y_{n}-p\right\|+\mu_{n}\right] \\
+\frac{\gamma_{n}}{\beta_{n}}\left[\nu_{n} M^{*}\left\|x_{n}-p\right\|+\mu_{n}\right] .
\end{gathered}
$$

From $\liminf \operatorname{in}_{n} \beta_{n}>0$, we get

$$
d=\lim _{n}\left\|x_{n}-p\right\| \leqslant \liminf _{n}\left\|z_{n}-p\right\| .
$$

This implies from (5) that

$$
\begin{aligned}
d & =\lim _{n}\left\|z_{n}-p\right\| \\
& =\lim _{n}\left\|a_{n}\left(T^{n} x_{n}-p\right)+\left(1-a_{n}\right)\left(x_{n}-p\right)\right\| .
\end{aligned}
$$

By the condition (ii), Lemma 3 and (8), we have

$$
\lim _{n}\left\|T^{n} x_{n}-x_{n}\right\|=0 .
$$


The conclusion $\lim _{n}\left\|x_{n}-T x_{n}\right\|=0$ can be obtained from Lemma 5 immediately. This completes the proof.

Recall that a Banach space $X$ is said to satisfy Opial's condition if $x_{n} \rightarrow x$ weakly and $x \neq y$ imply that

$$
\limsup _{n}\left\|x_{n}-x\right\| \leqslant \limsup _{n}\left\|x_{n}-y\right\| .
$$

Lemma 7 (Ref. 5) Let $X$ be a Banach space which satisfies Opial's condition and $\left\{x_{n}\right\}$ be a sequence in $X$. Let $u, v \in X$ be such that $\lim _{n}\left\|x_{n}-u\right\|$ and $\lim _{n}\left\|x_{n}-v\right\|$ exist. If $\left\{x_{n_{k}}\right\}$ and $\left\{x_{m_{k}}\right\}$ are subsequences of $\left\{x_{n}\right\}$ which converge weakly to $u$ and $v$, respectively, then $u=v$.

The next theorem follows closely from Chang's theorem ${ }^{9}$.

Theorem 1 Let $X$ be a uniformly convex Banach space and $C$ be a nonempty closed convex subset of $X, T: C \rightarrow C$ be a uniformly L-Lipschitzian and $\left(\left\{\mu_{n}\right\},\left\{\nu_{n}\right\}, \zeta\right)$-total asymptotically nonexpansive mapping. Then $I-T$ is demiclosed at 0 , i.e., $x_{n} \rightarrow x$ weakly and $x_{n}-T x_{n} \rightarrow 0$ strongly, then $x$ is a fixed point of $T$.

Theorem 2 Let $X$ be a uniformly convex Banach space which satisfies Opial's condition, $C$ be a nonempty closed convex subset of $X$, and $T: C \rightarrow C$ be a uniformly L-Lipschitzian and $\left(\left\{\mu_{n}\right\},\left\{\nu_{n}\right\}, \zeta\right)$ total asymptotically nonexpansive mapping. Let $\left\{x_{n}\right\}$ be a sequence in $C$ defined by Algorithm 1. Then $\left\{x_{n}\right\}$ converges weakly to a fixed point of $T$.

Proof: It follows from Lemma 6 that $\lim _{n}\left\|x_{n}-T x_{n}\right\|=0$. Since $X$ is uniformly convex and $\left\{x_{n}\right\}$ is bounded, without loss of generality, we may assume that $x_{n} \rightarrow u$ weakly. By Theorem 1, $u$ is a fixed point of $T$. Suppose that subsequences $\left\{x_{n_{k}}\right\}$ and $\left\{x_{n_{j}}\right\}$ of $\left\{x_{n}\right\}$ converge weakly to $u$ and $v$, respectively. From Theorem 1, $u$ and $v$ are fixed points of $T$. By Lemma $2, \lim _{n}\left\|x_{n}-u\right\|$ and $\lim _{n}\left\|x_{n}-v\right\|$ exist. It follows from Lemma 7 that $u=v$. Therefore $\left\{x_{n}\right\}$ converges weakly to a fixed point of $T$.

Theorem 3 Let $X$ be a uniformly convex Banach space, $C$ be a nonempty closed convex subset of $X$, and $T: C \rightarrow C$ be a uniformly L-Lipschitzian and $\left(\left\{\mu_{n}\right\},\left\{\nu_{n}\right\}, \zeta\right)$-total asymptotically nonexpansive mapping. Let $\left\{x_{n}\right\}$ be a sequence in $C$ defined by Algorithm 1. Then $\left\{x_{n}\right\}$ converges strongly to a fixed point of $T$.
Proof: Let $p$ be a fixed point of $T$. From Lemma 2, we know that $\lim _{n}\left\|x_{n}-p\right\|$ exists, then $\left\{x_{n}\right\}$ is bounded. By Lemma 6, we have

$$
\lim _{n}\left\|x_{n}-T x_{n}\right\|=0
$$

Since $T$ is completely continuous and $\left\{x_{n}\right\}$ is bounded, there exists a subsequence $\left\{x_{n_{k}}\right\}$ of $\left\{x_{n}\right\}$ such that $\left\{T x_{n_{k}}\right\}$ converges. Hence $\left\{x_{n_{k}}\right\}$ converges from (4). Let $\lim _{k} x_{n_{k}}=q$, by continuity of $T$ and (4) we have $T q=q$, so $q$ is a fixed point of $T$. It follows from Lemma 2 that $\lim _{n}\left\|x_{n}-q\right\|=0$.

Acknowledgements: The author would like to thank the referees for helpful comments. This work was supported by the Natural Science Foundation of CQ CSTC under Grant No. cstc2014jcyjA00022.

\section{REFERENCES}

1. Noor MA (2000) New approximation schemes for general variational inequalities. J Math Anal Appl 251, 217-29.

2. Glowinski R, Le Tallec P (1989) Augmented Lagrangian and Operator-Splitting Methods in Nonlinear Mechanics, SIAM, Philadelphia.

3. Haubruge S, Nguyen VH, Strodiot JJ (1998) Convergence analysis and applications of the Glowinski-Le Tallec splitting method for finding a zero of the sum of two maximal monotone operators. J Optim Theor Appl 97, 645-73.

4. Xu BL, Noor MA (2002) Fixed-point iterations for asymptotically nonexpansive mappings in Banach spaces. J Math Anal Appl 267, 444-53.

5. Suantai S (2005) Weak and strong convergence criteria of Noor iterations for asymptotically nonexpansive mappings. J Math Anal Appl 311, 506-17.

6. Nilsrakoo W, Saejung S (2006) A new three-step fixed point iteration scheme for asymptotically nonexpansive mappings. Appl Math Comput 181, 1026-34.

7. Nilsrakoo W, Saejung S (2007) A reconsideration on convergence of three-step iterations for asymptotically nonexpansive mappings. Appl Math Comput 190, 1472-8.

8. Geobel K, Kirk WA (1972) A fixed point theorem for asymptotically nonexpansive mappings. Proc Am Math Soc 35, 171-4.

9. Chang SS, Wang L, Joseph Lee HW, Chan CK, Yang L (2012) Demiclosed principle and $\Delta$-convergence theorems for total asymptotically nonexpansive mappings in CAT(0) spaces. Appl Math Comput 219, 2611-7.

10. Tan KK, Xu HK (1993) Approximating fxed points of nonexpansive mappings by the Ishikawa iteration process. J Math Anal Appl 178, 301-8. 